\title{
Influência do teor de glúten na qualidade de biscoitos elaborados com farinha de triticale
}

\author{
Érika Watanabe ${ }^{1}$, Klever Marcio Antunes Arruda ${ }^{2}$, Cintia Sorane Good Kitzberger ${ }^{3}$, Maria \\ Brigida dos Santos Scholz ${ }^{4}$ e Alexandre Rodrigo Coelho ${ }^{5}$
}

Triticale ( $x$ Triticosecale Wittmack) é um cereal com potencial uso industrial, desenvolvido como alternativa ao trigo. Apesar de combinar o rendimento e qualidade do trigo com a adaptabilidade ambiental e resistência às doenças do centeio, a baixa qualidade de glúten da farinha limita seu uso na indústria alimentícia. Nesse contexto, o presente estudo teve como objetivo avaliar a influência do conteúdo de glúten na qualidade de biscoitos confeccionados com farinha de triticale. Devido ao baixo teor de glúten (TLD 1202, não detectado e BRS Minotauro 11,35\%), as farinhas de triticale tiveram tempo de desenvolvimento reduzido (0,50 para TLD $1202 \mathrm{e}$ BRS Minotauro 1,10 minutos), baixa estabilidade da massa (0,40 para TLD 1202 e BRS Minotauro 3,00 minutos) e capacidade reduzida de retenção de ácido láctico (64,14\% para TLD 1202 e 78,20\% para BRS Minoauro). Contudo, essas farinhas influenciaram positivamente a largura, fator de expansão e textura instrumental dos biscoitos produzidos. Com índices de aceitação acima de $81 \%$, biscoitos com farinha de triticale superaram os biscoitos elaborados com farinha de trigo em todos os atributos sensoriais avaliados. As farinhas de triticale mostraram propriedades adequadas para fabricar de biscoitos de alta qualidade, mantendo as características do produto, independentemente do conteúdo de glúten.

Palavras-chave: Farinografia, capacidade de retenção de solventes, avaliação sensorial, textura.

\section{Influence of gluten content on the quality of cookies made with triticale flour}

Triticale ( $x$ Triticosecale Wittmack) is a cereal crop that has a potential industrial use as a wheat alternative. This cereal combines the yield and quality of wheat with the environmental adaptability and disease resistance of rye, but its low gluten quality limits the industrial use of flour in processed foods. In this context, the current study aimed to evaluate the influence of gluten content on the quality of cookies made with triticale flour. Due to the low gluten content (TLD 1202, undetected and BRS Minotauro 11.35\%), triticale flours showed a short development time (TLD 12020.50 and BRS Minotauro 1.10 minutes), low dough stability (TLD 1202 of 0.40 and BRS Minotauro 3.00 minutes) and reduced lactic acid retention capacity (64.14\% for TLD 1202 and 78.20\% for BRS Minoauro). However, these flours positively influenced the width, expansion factor and instrumental texture of produced cookies. With acceptance rates above $81 \%$, cookies made with triticale flours outperformed the cookies made with wheat flour in all sensory attributes analyzed. Triticale flours have shown suitable properties for the manufacture of high quality cookies, maintaining the characteristics of the product, regardless of the gluten content.

\footnotetext{
1 Tecnóloga em Alimentos, Mestre em Tecnologia de Alimentos, Universidade Tecnológica Federal do Paraná. Endereço para correspondência: Av. dos Pioneiros, 3131, Londrina, PR.CEP 86036-370. Telefone: (43) 3315-6153.E-mail: erikawatanabe@utfpr.edu.br.

${ }^{2}$ Pesquisador, Doutor em Fitotecnia, Instituto Agronômico do Paraná. Endereço para correspondência: Rod. Celso Garcia Cid, km 375, Londrina, PR. CEP 86047902. Telefone: (43) 33762374.E-mail:klever@iapar.br

${ }^{3}$ Pesquisadora, Doutora em Ciência de Alimentos, Instituto Agronômico do Paraná. Endereço para correspondência: Rod. Celso Garcia Cid, km 375, Londrina, PR. CEP 86047902. Telefone: (43) 33762374. E-mail: cintiasorane@yahoo.com.br

${ }^{4}$ Pesquisadora, Doutora em Ciência de Alimentos, Instituto Agronômico do Paraná. Endereço para correspondência: Rod. Celso Garcia Cid, km 375, Londrina, PR. CEP 86047902. Telefone: (43) 33762374.E-mail:mbscholz@iapar.br

${ }^{5}$ Professor, Doutor em Ciência de Alimentos, Universidade Tecnológica Feeral do Paraná. Endereço para correspondência: Av. dos Pioneiros, 3131, Londrina, PR.CEP 86036-370. Telefone: (43) 3315-6153.E-mail: arcoelho@utfpr.edu.br. https://orcid.org/0000-0001-5744-5666.
} 
Keywords: Farinograph, solvent retention capacity, sensory evaluation, texture.

\section{INTRODUÇÃO E OBJETIVOS}

O triticale hexaploide ( $\mathrm{x}$ Triticosecale Wittmack) é um cereal híbrido que possui os genomas A e B do trigo tetraploide (Triticum durum) e o genoma $\mathrm{R}$ do centeio (Secale cereale L.). Este cereal foi produzido com o intuito de combinar o rendimento e a qualidade do trigo com a adaptabilidade ambiental e a resistência à doença do centeio [1].

Em condições de seca, degradação da terra e mudanças climáticas que restringem a produção de trigo, o triticale é uma cultura alternativa que pode auxiliar a aumentar a oferta de alimentos. Além disso, contribui para que a indústria alimentícia possa utilizar novos ingredientes $\mathrm{e}$ diversificar sua produção [2,3].

O triticale apresenta ainda outras vantagens em relação ao trigo, tais como melhor balanço mineral, maiores teores de fibras solúveis e lisina (o primeiro aminoácido limitante em cereais), além da presença de compostos fenólicos com atividade antioxidante (ácido ferúlico, proantocianidinas e lignanas) $[4,5,6]$.

Entretanto, o uso da farinha de triticale em alimentos processados ainda é limitado. A cor escura da farinha, a alta atividade de $\alpha$-amilase e reduzida força de glúten inviabilizam sua utilização em produtos fermentados [7].

As propriedades únicas da farinha de trigo comum (Triticum aestivum, genoma AABBDD) para a fabricação de pães levedados se deve à capacidade de suas proteínas de reserva em formar gluten [8]. Genes localizados no genoma D do trigo são responsáveis por codificar tais proteínas. Para o triticale hexaploide (genoma AABBRR), além da ausência do genoma $\mathrm{D}$, a presença do genoma $\mathrm{R}$ herdado do centeio (que não tem a capacidade de formar glúten), resulta em uma qualidade de panificação inferior [9]. Entretanto, a indústria alimentícia nem sempre necessita de farinhas com alto teor de glúten. Para cada produto final ou para cada processo mecanizado definido, existe uma farinha com características específicas.
Com base no exposto, o objetivo deste estudo foi avaliar a influência do teor de glúten sobre a qualidade dos cookies elaborados com farinha de triticale.

\section{MATERIAL E MÉTODOS}

Foram avaliados dois genótipos de triticale brasileiros (cultivar BRS Minotauro e linhagem TLD 1202) e um genótipo de trigo hexaploide (linhagem LD 122105). Cada amostra consistiu de 4 $\mathrm{kg}$ de grãos, cedidos pelo Instituto Agronômico do Paraná (IAPAR) e colhidos no ano de 2014.

\section{Extração e análise da farinha}

A extração das farinhas foi realizada em moinho experimental Chopin (modelo CD1), seguindo os métodos 26-10 e 16-10 da American Association of Cereal Chemists - AACC, com ajuste prévio da umidade dos grãos a $15,5 \%$ [10]. O teor de umidade foi avaliado pelo método 44-15A [10].

O teor de proteína bruta foi determinado pelo método 46-13, empregando-se o valor de 5,7 como fator de conversão do nitrogênio total para proteína e o conteúdo de cinzas foi determinado de acordo com o método 08-03.01 [10]. A quatificação do glúten foi realizada por meio dos métodos 38-10 e 38-12A10, no Sistema Glutomatic (Perten Instrument ${ }^{[10]}$. Os componentes cromáticos de luminosidade $\left(\mathrm{L}^{*}\right)$, vermelho verde $\left(\mathrm{a}^{*}\right)$ e azulamarelo (b*) foram determinados com auxílio de colorímetro Minolta Chroma Meter 410C.

As propriedades de mistura da massa: absorção de água, tempo de desenvolvimento e estabilidade foram avaliadas por meio da farinografia, utilizando o Farinógrafo Brabender, de acordo com o método 54-21 [10]. A capacidade de retenção de solvente (CRS) foi realizada com quatro solventes: água, sacarose $50 \%$, carbonato de sódio 5\% e ácido láctico 5\% segundo o método 5611 [10], com adaptação de Bettge [11].

\section{Formulação dos cookies}


Os cookies foram elaborados com três tipos de farinha, sendo duas de triticale e uma de trigo (controle), totalizando três formulações.

A formulação controle consistiu de $1200 \mathrm{~g}$ de farinha de trigo (LD 122105), $270 \mathrm{~g}$ de açúcar granulado, $270 \mathrm{~g}$ de açúcar mascavo, $450 \mathrm{~g}$ de manteiga, $260 \mathrm{~g}$ de ovos, $9 \mathrm{~g}$ de fermento em pó, $9 \mathrm{~g}$ de cloreto de sódio e $600 \mathrm{~g}$ de gotas de chocolate meio amargo. Para a elaboração dos cookies de triticale, a farinha de trigo foi substituída pelas farinhas de triticale em outras duas elaborações.

Para a preparação da massa, o açúcar e a manteiga foram misturados até obtenção de um creme leve, seguido de adição dos demais ingredientes na seguinte ordem: ovos, cloreto de sódio, fermento em pó e gotas de chocolate. Porções de aproximadamente 14,6 g de massa foram modeladas e assadas em forno convencional a $180^{\circ} \mathrm{C}$ durante 15 minutos.

\section{Composição centesimal dos cookies}

Para a determinação dos teores de umidade, cinzas e proteína, foram utilizados os mesmos métodos aplicados para as farinhas, com valor de 6,25 como fator de conversão de nitrogênio total para proteína bruta ${ }^{[10]}$. O conteúdo lipídico foi determinado de acordo com o método 2003.6, recomendado pela Associação de Química Analítica Oficial - AOAC ${ }^{[2]}$ e o carboidrato, quantificado por subtração.

\section{Propriedades físicas dos cookies}

A análise de cor dos biscoitos foi realizada com auxílio do colorímetro Minolta Chroma Meter 410C, determinando-se os seguintes componentes cromáticos: luminosidade $\left(\mathrm{L}^{*}\right)$, vermelho verde $\left(\mathrm{a}^{*}\right)$ e azul-amarelo ( $b^{*}$ ). Os parâmetros peso (antes e após o cozimento), largura e espessura foram determinados pelo método 10-50D [10]. O fator de expansão foi calculado com base na relação entre a largura e a espessura do biscoito, ou seja, quanto maior o fator de expansão, melhor a qualidade dos cookies.

O texturômetro TA.XT plus (Stable Micro Systems) foi empregado para medir a dureza (expressa em Newton) e a fraturabilidade (em milímetros) dos cookies. O probe utilizado foi o HDP / 3PB e o ensaio realizado nas seguintes condições: velocidade de pré-teste $1,0 \mathrm{~mm} / \mathrm{s}$, velocidade de teste $3,0 \mathrm{~mm} / \mathrm{s}$, velocidade pós-teste $10,0 \mathrm{~mm} / \mathrm{s}$, distância $4,0 \mathrm{~mm}$ e força $0,5 \mathrm{~N}$.

\section{Avaliação sensorial}

O painel sensorial foi constituído de oitenta provadores não treinados, recrutados entre funcionários e estudantes da Universidade Tecnológica Federal do Paraná - UTFPR, Campus Londrina. Os provadores, de ambos os sexos, eram maiores de 18 anos de idade e não possuíam alergia a nenhum ingrediente utilizado na formulação dos cookies. Para análise, os pesquisadores forneceram um cookie de cada formulação, instruindo os participantes a avaliar a cor, sabor, textura, aroma e impressão geral. Os provadores indicaram a resposta para cada atributo sensorial em uma escala hedônica híbrida de 10 pontos, ancorada em três pontos: $0=$ "desgostei extremamente", $5=$ "nem gostei nem desgostei" e 10 = "gostei extremamente" [13]. O índice de aceitabilidade dos cookies foi determinado com base na metodologia proposta por Dutcosky [14], onde uma percentagem maior ou igual a 70\% indica uma boa aceitação do produto. A avaliação sensorial foi previamente aprovada pelo Comitê de Ética em Pesquisa, da Universidade Tecnológica Federal do Paraná (Certificado de Apresentação para Avaliação Ética No. 53604116.5.0000.5547).

\section{Análise estatística}

Os dados das análises físico-químicas das amostras de farinha e biscoito, assim como os resultados das propriedades físicas e sensoriais dos cookies foram submetidos à análise de variância (ANOVA) e as médias comparadas pelo teste de Tukey a 5\% de probabilidade, pelo programa Statistica versão 7.0.

\section{RESULTADOS E DISCUSSÃO}

As farinhas extraídas das amostras de triticale BRS Minotauro e TLD 1202 apresentaram menor teor de proteínas do que a farinha de trigo (Tabela 1).

O conteúdo de proteínas recomendado em farinhas para fabricação de biscoitos situa-se entre 70,00 e 120,00 g. $\mathrm{kg}^{-1}$, pois níveis mais altos podem causar miolo de textura áspera e rachaduras na superfície dos cookies [15]. Sendo assim, o conteúdo 
protéico encontrado nas farinhas de triticale foi considerado satisfatório. Embora a farinha de trigo tenha apresentado teor de proteína acima do ideal para o produto, as indústrias costumam misturar a farinha com amido, a fim de diluir o excesso de proteínas.

A farinha do triticale BRS Minotauro apresentou o maior teor de cinzas $\left(8,80 \mathrm{~g}^{\mathrm{kg}} \mathrm{kg}^{-1}\right)$ e consequentemente, sua cor foi a mais escura. Por outro lado, o teor de cinzas e a cor da farinha do triticale TLD 1202 não diferiu do trigo controle $(p>$ $0,05)$, mostrando avanços nas características dos genótipos de triticale modernos. No início do cultivo de triticale, a farinha resultante do processamento era escura e como os consumidores valorizavam farinhas de cor branca e brilhante, havia pouca aceitação comercial para as farinhas de triticale [3,7]. Entretanto, a procura por alimentos mais saudáveis vem aumentando o consumo de produtos integrais e consequentemente, existe uma maior aceitação de farinhas de cor escura pelos consumidores atuais [16].

Tabela 1. Propriedades físico-químicas das farinhas extraídas de triticales brasileiros (cultivar BRS Minotauro e linhagem TLD 1202) e um genótipo de trigo hexaploide (linhagem LD 122105).

\begin{tabular}{|c|c|c|c|}
\hline \multirow[t]{2}{*}{ Parâmetro } & \multicolumn{3}{|c|}{ Genótipo } \\
\hline & $\begin{array}{c}\text { BRS } \\
\text { Minotauro }\end{array}$ & TLD 1202 & LD 122105 \\
\hline $\begin{array}{l}\text { Proteína } \\
\left(\mathrm{g} \cdot \mathrm{kg}^{-1}\right)\end{array}$ & $111,30^{b} \pm 0,30$ & $95,10^{c} \pm 0,01$ & $137,00^{a} \pm 0,25$ \\
\hline $\begin{array}{l}\text { Cinzas } \\
\left(\mathrm{g} \cdot \mathrm{kg}^{-1}\right)\end{array}$ & $0,88^{a} \pm 0,04$ & $0,69^{\mathrm{b}} \pm 0,04$ & $0,66^{\mathrm{b}} \pm 0,01$ \\
\hline $\mathrm{L}^{*}$ & $91,30^{\mathrm{b}} \pm 0,03$ & $92,85^{a} \pm 0,11$ & $92,79 a \pm 0,03$ \\
\hline$a^{*}$ & $0,16 \mathrm{a} \pm 0,01$ & $-0,30^{\mathrm{b}} \pm 0,01$ & $-0,87 \mathrm{c} \pm 0,01$ \\
\hline$b^{*}$ & $6,35^{b} \pm 0,02$ & $6,45 \mathrm{~b} \pm 0,04$ & $6,68^{a} \pm 0,02$ \\
\hline
\end{tabular}

De acordo com a Tabela 2, o teor de glúten da farinha de triticale Minotauro BRS foi menor que o da farinha de trigo (controle). Para a amostra de triticale TLD 1202, não foi possível detectar o conteúdo de gluten pelo método utilizado.

O glúten resulta da interação entre as proteínas de reserva, gliadinas e gluteninas, que conferem propriedades de elasticidade (resistência) e extensibilidade (viscosidade) da massa [7]. Muitos dos genes responsáveis pela formação de glúten estão localizados no genoma $\mathrm{D}$ do trigo hexaploide
(AABBDD). Tendo em vista que o triticale é originado do cruzamento entre trigo duro tetraploide (AABB) e do centeio diploide (RR), o triticale hexaploide (AABBRR) não possui o genoma D ${ }^{[17]}$.

Apesar da tolerância ao estresse abiótico e ao maior potencial de produção conferido pelas espécies parentais, a ausência do genoma $\mathrm{D}$ reduz a formação de glúten do triticale e, consequentemente, sua qualidade de panificação é inferior à do trigo hexaploide [17]. No entanto, considerando que as indústrias de processamento têm exigências de farinhas específicas para cada produto desenvolvido, a farinha de triticale pode ser aplicada em produtos que não requerem alta resistência ao glúten, como bolos e biscoitos ou podem ser misturados com trigo para fazer tortilhas ou em alimentos integrais ${ }^{[8,18]}$.

Tabela 2. Propriedades tecnológicas das farinhas extraídas de triticales brasileiros (cultivar BRS Minotauro e linhagem TLD 1202) e um genótipo de trigo hexaploide (linhagem LD 122105).

\begin{tabular}{|c|c|c|c|c|}
\hline \multirow{3}{*}{$\begin{array}{c}\text { Glúten } \\
(\%)\end{array}$} & & $\begin{array}{c}\text { BRS } \\
\text { Minotauro }\end{array}$ & TLD 1202 & LD 122105 \\
\hline & Úmido $^{1}$ & $11,35 \pm 0,56$ & ND & $29,74 \pm 1,13$ \\
\hline & $\mathrm{Seco}^{1}$ & $3,78 \pm 0,25$ & ND & $9,98 \pm 1,00$ \\
\hline \multirow{3}{*}{ Farino $^{2}$} & $\begin{array}{l}\text { Absorção de } \\
\text { água }(\%)^{1}\end{array}$ & $54,15^{a} \pm 0,35$ & $55,45^{a} \pm 0,35$ & $50,95^{\mathrm{b}} \pm 0,35$ \\
\hline & $\begin{array}{l}\text { Tempo de } \\
\text { desenvol. } \\
(\min )^{1}\end{array}$ & $1,10^{\mathrm{b}} \pm 0,14$ & $0,50^{\mathrm{b}} \pm 0,00$ & $17,90^{\mathrm{a}} \pm 4,10$ \\
\hline & $\begin{array}{c}\text { Estabilidade } \\
\quad(\min )^{1}\end{array}$ & $3,00^{\mathrm{b}} \pm 2,97$ & $0,40^{\mathrm{b}} \pm 0,00$ & $24,95^{a} \pm 5,59$ \\
\hline \multirow{4}{*}{$\begin{array}{l}\text { CRS } \\
(\%)\end{array}$} & Água ${ }^{1}$ & $59,51^{a} \pm 0,81$ & $58,18^{a} \pm 0,026$ & $59,96^{a} \pm 0,41$ \\
\hline & $\begin{array}{l}\text { Carbonato de } \\
\text { sódio }^{1}\end{array}$ & $77,36^{\mathrm{b}} \pm 1,08$ & $76,98^{b} \pm 0,22$ & $85,04^{a} \pm 0,11$ \\
\hline & Sacarose $^{1}$ & $90,55^{b} \pm 0,94$ & $88,73^{\mathrm{b}} \pm 0,90$ & $122,07^{a} \pm 0,51$ \\
\hline & $\begin{array}{l}\text { Ácido } \\
\text { láctico }{ }^{1}\end{array}$ & $78,20^{\mathrm{b}} \pm 0,10$ & $64,14 \mathrm{c} \pm 0,75$ & $159,81^{a} \pm 0,72$ \\
\hline
\end{tabular}

${ }^{1}$ Média \pm desvio padrão de duplicata. ${ }^{2}$ Farino $=$ farinográfia. $\mathrm{ND}=$ não detectado. Letras iguais na mesma linha não diferem estatisticamente, ao nível de $5 \%$ de significância, pelo teste de Tukey.

O tempo de desenvolvimento de massa para as farinhas de triticale foi significativamente menor que para a farinha de trigo. De forma análoga, a estabilidade das massas de triticale foi muito menor do que para a farinha controle $(p<$ $0,05)$. Assim, a massa obtida com a farinha de 
Qualidade instrumental e sensorial de cookies com triticale. Watanabe et al.

triticale atinge a consistência ótima rapidamente, mas perde a estabilidade com a mesma velocidade.

Aprodu e colaboradores [19] verificaram, igualmente, que massas obtidas de farinhas de triticale apresentavam menor tempo de desenvolvimento e estabilidade quando comparadas às farinhas de trigo. Ambos os parâmetros refletem a capacidade da farinha de formar glúten: o tempo de desenvolvimento está associado à qualidade das farinhas, ou seja, aquelas mais fortes apresentam maior tempo de desenolvimento, enquanto a estabilidade fornece a medida da tolerância da massa ao processo de mistura (maior tempo indica maior tolerância) [20]. Portanto, os resultados sugerem que a qualidade do glúten das farinhas de triticale foi inferior ao trigo.

Ainda em relação à Tabela 2 , a absorção de água determinada pelo Farinógrafo foi maior para as farinhas de triticale. A baixa absorção de água (50 a $54 \%$ para o padrão britânico) é uma característica desejável para as farinhas destinadas à fabricação de biscoitos, pois conferem baixa umidade, aumentam a vida útil e reduzem o tempo de forneamento do produto [21, 22]. Desta forma, a farinha de trigo exibiu a absorção de água própria para a produção de biscoitos (50,95\%), enquanto a absorção das farinhas TLD 1202 e BRS Minotauro estavam ligeiramente acima do ideal (55,46 e 54,15\%, respectivamente).

Para o teste de CRS, as três farinhas analisadas apresentaram capacidade de retenção de água semelhante. Contudo, a capacidade de retenção de ácido láctico, carbonato de sódio e sacarose das farinhas de triticale foram inferiores ao do trigo. $\mathrm{Na}$ avaliação da CRS, a capacidade de retenção de água está associada a todos os constituintes da farinha que têm capacidade de absorver água, enquanto o carbonato de sódio está relacionado ao teor de amido danificado, a sacarose com a concentração de pentosanas e gliadinas e o ácido láctico, por sua vez, é um indicador da qualidade de glúten [23]. Portanto, o resultado da CRS de ácido láctico corrobora com o obtido na análise quantitativa de glúten, indicando baixo teor de glúten para as farinhas de triticale. Observando a CRS de carbonato de sódio e de sacarose, verifica-se que as farinhas de triticale apresentaram reduzido teor de amido danificado, assim como pentosanas e gliadinas, quando comparadas à farinha de trigo.
Para produção de pães, exige-se que a farinha tenha alta absorção de água, alta resistência ao glúten e um conteúdo relativamente alto de amido danificado e pentosanas, enquanto a produção de biscoitos exige farinhas com baixa absorção de água, mínima resistência ao glúten e baixo conteúdo de amido danificado e pentosanas [23]. Desta forma, os resultados do teste de CRS sugerem que as farinhas de triticale seriam adequadas à produção de biscoitos.

A Tabela 3 apresenta a composição centesimal dos cookies. Todas as formulações apresentaram baixo teor de umidade, característica importante para os cookies, uma vez que valores inferiores a $50 \mathrm{~g} \cdot \mathrm{kg}^{-1}$ contribuem para a estabilidade do produto durante a sua vida útil [24].

O controle apresentou maior teor de proteínas: 73,10 g. $\mathrm{kg}^{-1}$, enquanto os biscoitos feitos com as farinhas de triticale apresentaram $65,70 \mathrm{~g} \cdot \mathrm{kg}^{-1}$ para o BRS Minotauro e 59,50 para o TLD 1202. Por outro lado, não houve diferença significativa $(p>0,05)$ nos teores de cinzas, lipídeos e carboidratos das três formulações. A semelhança nos teores de cinzas entre trigo e triticale indica avanços dos programas de melhoramento genético de triticale, cujo objetivo é desenvolver genótipos com características cada vez mais próximas ao trigo.

Para os parâmetros peso (antes e após o forneamento) e espessura, as formulações feitas com farinhas de triticale não diferiram do controle (Tabela 4). Os cookies elaborados com trigo apresentaram largura reduzida e, consequentemente, o fator de expansão desta formulação foi um pouco menor. Tal fato pode ser explicado pela quantidade de glúten presente na farinha. Altos níveis de gordura e açúcar na massa do cookie impedem a hidratação das proteínas e, consequentemente, não há desenvolvimento de glúten. No entanto, durante o processo de forneamento, o calor promove a solubilização dos açúcares e as proteínas formadoras de glúten tornam-se móveis, reagindo com a água disponível no sistema. Assim, a rede de glúten formada impede a propagação da massa e aumenta a resistência ao colapso, determinando a espessura e largura dos cookies [25]. Singh e colaboradores [26] substituíram a farinha de trigo por mistura de proteína de soja e glúten na elaboração de cookies e observaram que o teor de proteínas estava negativamente correlacionado com a largura e positivamente com a espessura, reduzindo o fator 
de expansão dos cookies. Além disso, observaram também que quanto maior o teor de glúten, maior era o percentual de redução da largura.

Tabela 3. Composição centesimal dos cookies produzidos com farinhas de triticales brasileiros (cultivar BRS Minotauro e linhagem TLD 1202) e um genótipo de trigo hexaploide (linhagem LD 122105).

\begin{tabular}{lccc}
\hline Componente & BRS Minotauro & TLD 1202 & LD 122105 \\
\hline Umidade & & & \\
$\left(\mathrm{g} \cdot \mathrm{kg}^{-1}\right)$ & $35,20^{\mathrm{a}} \pm 0,11$ & $32,50^{\mathrm{a}} \pm 0,09$ & $28,60^{\mathrm{a}} \pm 0,46$ \\
Proteina & & & \\
$\left(\mathrm{g} \cdot \mathrm{kg}^{-1}\right)$ & $65,70^{\mathrm{b}} \pm 0,11$ & $59,50^{\mathrm{c}} \pm 0,06$ & $73,10^{\mathrm{a}} \pm 0,11$ \\
Cinzas & & & \\
$\left(\mathrm{g} \cdot \mathrm{kg}^{-1}\right)$ & $13,56^{\mathrm{a}} \pm 0,02$ & $13,52^{\mathrm{a}} \pm 0,08$ & $13,38^{\mathrm{a}} \pm 0,02$ \\
$\mathrm{Lipidios}^{-}$ & & & \\
$\left(\mathrm{g} \cdot \mathrm{kg}^{-1}\right)$ & $208,13^{\mathrm{a}} \pm 1,46$ & $216,11^{\mathrm{a}} \pm 0,02$ & $214,35^{\mathrm{a}} \pm 0,34$ \\
Carboidrato & & & \\
$\left(\mathrm{g} \cdot \mathrm{kg}^{-1}\right)$ & 677,41 & 678,37 & 670,57 \\
\hline $\begin{array}{l}\text { Média } \pm \text { desvio padrão, de duplicata; valores expressos em base seca. } \\
\text { Letras iguais na mesma linha não diferem estatisticamente, ao nível de } 5 \% \text { de } \\
\text { significância, pelo teste de Tukey. }\end{array}$
\end{tabular}

Os cookies produzidos com a farinha do triticale TLD1202 exibiram cor mais clara para a análise instrumental (Tabela 4), indicando que a cor da farinha não influenciou o produto final, uma vez que o triticale TLD 1202 e trigo não apresentaram diferenças na coloração da farinha (Tabela 1). O desenvolvimento de cor ocorre devido ao efeito da reação entre açúcares redutores e aminoácidos (reação de Maillard) ou dextrinização do amido e caramelização dos açúcares [27]. A cor mais clara dos cookies produzidos com a farinha de triticale TLD 1202 ocorreu, portanto, devido ao reduzido teor de proteínas, o que restringiu a disponibilidade de aminoácidos para a reação de Maillard. Singh e colaboradores [26] avaliaram a cor de cookies com proteína de soja e observaram que o aumento no teor de proteínas da formulação resultou em um produto de cor mais escura.

O reduzido teor de glúten das farinhas de triticale influenciou positivamente na textura instrumental dos biscoitos, reduzindo sua dureza e fraturabilidade (Tabela 4). Colussi e colaboradores [28] também observaram que a substituição da farinha de trigo por farinha de arroz ocasionou redução da dureza e fraturabilidade dos biscoitos, devido à ausência de glúten na farinha de arroz.

Tabela 4. Propriedades físicas dos cookies elaborados com farinhas de triticales brasileiros (BRS Minotaure; TLD 1202) e de trigo (LD 122105).

\begin{tabular}{lccc}
\hline Parâmetro & $\begin{array}{c}\text { BRS } \\
\text { Minotauro }\end{array}$ & TLD 1202 & LD 122105 \\
\hline Peso antes do & $14,63^{\mathrm{a}} \pm 0,02$ & $14,64^{\mathrm{a}} \pm 0,01$ & $14,64^{\mathrm{a}} \pm 0,02$ \\
forneamento $^{1}(\mathrm{~g})$ & & & \\
Peso após & & & \\
forneamento $^{1}(\mathrm{~g})$ & $13,24^{\mathrm{a}} \pm 0,11$ & $13,17^{\mathrm{a}} \pm 0,08$ & $13,26^{\mathrm{a}} \pm 0,09$ \\
Largura $^{1}(\mathrm{~mm})$ & $51,08^{\mathrm{a}} \pm 0,36$ & $51,27^{\mathrm{a}} \pm 0,81$ & $48,34^{\mathrm{b}} \pm 0,80$ \\
Espessura $^{1}(\mathrm{~mm})$ & $15,68^{\mathrm{a}} \pm 0,41$ & $15,47^{\mathrm{a}} \pm 0,28$ & $15,48^{\mathrm{a}} \pm 0,44$ \\
Fator de & $3,26^{\mathrm{a}} \pm 0,07$ & $3,32^{\mathrm{a}} \pm 0,06$ & $3,13^{\mathrm{b}} \pm 0,08$ \\
expansão $^{1}$ & $55,80^{\mathrm{b}} \pm 1,10$ & $57,77^{\mathrm{a}} \pm 1,01$ & $55,69^{\mathrm{b}} \pm 0,58$ \\
L*1 $^{* 1}$ & $5,86^{\mathrm{a}} \pm 0,47$ & $5,94^{\mathrm{a}} \pm 0,83$ & $5,91^{\mathrm{a}} \pm 0,56$ \\
$\mathrm{a}^{* 1}$ & $35,01^{\mathrm{a}} \pm 0,41$ & $35,44^{\mathrm{b}} \pm 0,94$ & $33,79^{\mathrm{a}} \pm 0,41$ \\
$\mathrm{~b}^{* 1}$ & $60,62^{\mathrm{b}} \pm 2,36$ & $54,86^{\mathrm{b}} \pm 3,28$ & $74,77^{\mathrm{a}} \pm 4,85$ \\
Dureza $\left.^{(\mathrm{N})}\right)^{2}$ & & & \\
Fraturabilidade & $0,81^{\mathrm{b}} \pm 0,12$ & $0,87^{\mathrm{b}} \pm 0,10$ & $1,20^{\mathrm{a}} \pm 0,14$ \\
(mm $^{2}$ & & & \\
\hline
\end{tabular}

${ }^{1}$ Média \pm desvio padrão, de cinco respostas. ${ }^{2}$ Média \pm desvio padrão, de dez respostas. Letras iguais na mesma linha não diferem estatisticamente, ao nível de $5 \%$ de significância, pelo teste de Tukey.

Em relação ao teste sensorial, os cookies elaborados com as farinhas de triticale apresentaram a melhor textura. $\mathrm{Na}$ avaliação instrumental, a formulação controle apresentou maior dureza e fraturabilidade. Entretanto, sensorialmente os avaliadores não detectaram diferenças entre o cookie controle e aqueles elaborados com farinha de triticale TLD 1202 (Tabela 5).

Quanto ao parâmetro cor, embora a análise instrumental tenha indicado coloração mais clara para a formulação elaborada com a farinha de triticale TLD 1202 (Tabela 4), os provadores não detectaram essa diferença na avaliação sensorial $(p>$ $0,05)$ (Tabela 5).

Para os atributos aroma e sabor, os cookies produzidos com farinha de triticale TLD 1202 alcançaram pontuações mais elevadas que $\mathrm{O}$ controle, provavelmente devido ao sabor amendoado e naturalmente doce do triticale. Não obstante, o atributo impressão global se destacou 
nas amostras elaboradas com triticale, quando comparadas ao controle (Tabela 5).

Tabela 5. Análise sensorial de cookies elaborados com farinhas de triticales brasileiros (BRS Minotaure; TLD 1202) e de trigo (LD 122105).

\begin{tabular}{|c|c|c|c|}
\hline & BRS Minotauro & TLD 1202 & $\begin{array}{c}\text { LD } \\
122105\end{array}$ \\
\hline Cor & $8,01^{a} \pm 1,65$ & $8,29 a \pm 1,55$ & $7,83^{\mathrm{a}} \pm 1,58$ \\
\hline Aroma & $7,90^{a b} \pm 1,42$ & $8,30^{a} \pm 1,49$ & $7,69 \mathrm{~b} \pm 1,69$ \\
\hline Sabor & $8,36^{a b} \pm 1,41$ & $8,55^{a} \pm 1,33$ & $7,96^{\mathrm{b}} \pm 1,51$ \\
\hline Textura & $8,21 \mathrm{a} \pm 1,65$ & $7,62^{\mathrm{ab}} \pm 1,99$ & $7,16^{\mathrm{b}} \pm 2,02$ \\
\hline $\begin{array}{l}\text { Impressão } \\
\text { Global }\end{array}$ & $8,40^{a} \pm 1,15$ & $8,38^{\mathrm{a}} \pm 1,10$ & $7,77^{b} \pm 1,40$ \\
\hline $\begin{array}{l}\text { Índice de } \\
\text { Aceitação }(\%)\end{array}$ & 81,82 & 82,30 & 76,87 \\
\hline
\end{tabular}

A Figura 1 permite uma melhor visualização dos escores médios das três formulações para cada atributo sensorial e indica a preferência dos provadores pelos cookies elaborados com as farinhas de triticale. Para os cookies derivados do triticale BRS Minotauro, a textura foi o atributo mais relevante, enquanto aqueles produzidos a partir do triticale TLD 1202 se destacaram pelos atributos cor, aroma e sabor.

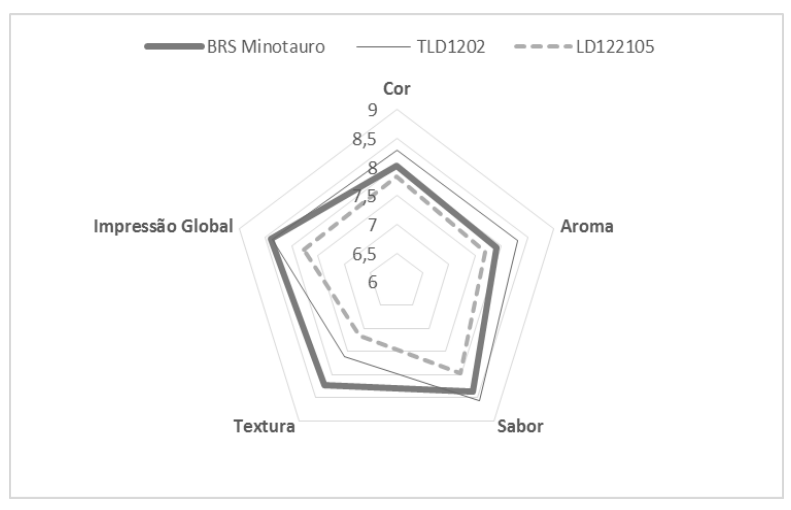

Figura 1. Aceitação dos cookies em relação aos atributos avaliados.

\section{CONCLUSÃO}

As farinhas de triticale foram adequadas para a produção de cookies, resultando em um maior fator de expansão, melhor textura, menor dureza e menor fraturabilidade.

$\mathrm{Na}$ avaliação sensorial, apesar das três formulações terem sido bem aceitas, os cookies elaborados com as farinhas de triticale atingiram as melhores pontuações. Desta forma, foi possível produzir cookies com farinhas de triticale mantendo as características do produto, independentemente da quantidade de glúten presente na farinha.

\section{AGRADECIMENTOS}

Os autores agradecem o IAPAR e Moinho Globo, pelas análises das farinhas, UEL pelas análises físicas do biscoito, e a UTFPR, pelas análises sensoriais e apoio financeiro.

\section{REFERÊNCIAS}

[1] Randhawa HS, Bona L, Graf RJ. Triticale breeding-progress and prospect. In: Eudes F. (Ed.). Triticale. Springer: Switzerland, 2015.

[2] Oliete B, Pérez GT, Gómez M, Ribotta PD, Moiraghi M, León AE. Use of wheat, triticale and rye flours in layer cake production. International Journal of Food Science and Technology. 2010; 45:697-706.

[3] Pattison AL \& Trethowan RM. Characteristics of modern triticale quality: commercially significant flour traits and cookie quality. Crop Pasture Science. 2013; 64: 874-880.

[4] Hosseinian FS \& Mazza G. Triticale bran and straw: potential new sources of phenolic acids, proanthocyanidins, and lignans. Journal of Functional Foods. 2009: 1: 57-64.

[5] Rakha A, Åman P, Andersson R. Dietary fiber in triticale grain: variation in content, composition, and molecular weight distribution of extractable components. Journal of Cereal Science. 2011; 54:324-331.

[6] Arendt EK \& Zannini E. Triticale. In: Arendt EK \& Zannini E (Eds). Cereal Grains for the Food and Beverage Industries. Woodhead Publishing: Cambridge, UK; 2013.

[7] Peña RJ. Food uses of triticale. In: Mergoum M \& GómezMacpherson H (Eds). Triticale Improvement and Production, FAO Plant Production and Protection Paper no 179. Food and Agricultural Organization of the United Nations, Rome; 2004. 
Qualidade instrumental e sensorial de cookies com triticale. Watanabe et al.

[8] Coşkuner Y \& Karababa E. Studies on the quality of Turkish flat breads based on blends of triticale and wheat flour. International Journal of Food Science and Technology. 2005; 40: 469-479.

[9] Martinek P, Vinterová M, Burešová I, Vyhnánek T. Agronomic and quality characteristics of triticale (X Triticosecale Wittmack) with HMW glutenin subunits 5+10. Journal of Cereal Science. 2008; 47:68-78.

[10] American Association of Cereal Chemists - AACC. Approved Methods of the American Association of Cereal Chemists. AACC International, 10th ed. St. Paul: MN, USA. 2000.

[11] Bettge AD, Morris CF, DeMacon VL, Kidwell KK. Adaptation of AACC method 56-11, solvent retention capacity, for use as an early generation selection tool for cultivar development. Cereal Chemistry. 2002; 79:670-674.

[12] Associação de Química Analítica Oficial - AOAC. Official method 2003.06 crude fat in feeds, cereal grains, and forages. Journal of AOAC International. 86: 888-899. 2003.

[13] Villanueva NDM, Petenate AJ, Da Silva MAAP. Performance of the hybrid hedonic scale as compared to the traditional hedonic, self-adjusting and ranking scales. Food Qual Prefer. 2005; 16: 691-703.

[14] Dutcosky S.D. Análise Sensorial de Alimentos. Champagnat: Curitiba, Brazil; 2007.

[15] Suas M. Cookies. In: Suas M. (Ed.). Advanced Bread and Pastry: a Professional Approach. Delmar Cengage Learning: Clifton Park, USA; 2008.

[16] Fohner GR \& Hernández-Sierra A. Triticale marketing: strategies for matching crop capabilities to user needs. In: Mergoum M \& Gómez-Macpherson H (Eds). Triticale Improvement and Production, FAO Plant Production and Protection Paper no 179. Food and Agricultural Organization of the United Nations, Rome; 2004.

[17] Tohver M, Kann A, Täht R, Mihhalevski A, Hakman J. Quality of triticale cultivars suitable for growing and breadmaking in northern conditions. Food Chemistry. 2005: 89: 125132.

[18] Nascimento Junior A, Baier AC, Teixeira MCC, Wiethölter S. Triticale in Brazil. In: Mergoum M \& Gómez-Macpherson H. (Eds). Triticale Improvement and Production, FAO Plant Production and Protection Paper no 179. Food and Agricultural Organization of the United Nations, Rome; 2004.

[19] Aprodu I \& Banu I. Comparative analyses of physicochemical and technological properties of triticale, rye and wheat. Ann Univ Dunarea Jos Galati Fasc. 2016; 40:31-39.
[20] Czank B, Ács P, Kovács ZS, Czankné CS, Bóna L. The puff-pastry making properties of triticale flour. Analecta. 2014; 1:81-84.

[21] Cauvain SP \& Young, LS. Tecnologia de Panificação, 2nd ed. Manole, Barueri, Brazil; 2009.

[22] Cunha GR \& Caierão E. Informações Técnicas para Trigo e Triticale - Safra 2015, Embrapa; 2014. Citado em 10 de abril de 2019. Disponível em: https://www.embrapa.br/documents/1355291/1729833/2015 -inf +tecn +trigo +e+triticale.pdf/205d3919-c572-4410-bc4d$\underline{1499 \mathrm{~b} 94333 \mathrm{ba}}$

[23] Kweon M, Slade L, Levine H. Solvent retention capacity (SRC) testing of wheat flour: principles and value in predicting flour functionality in different wheat-based food processes and in wheat breeding: a review. Cereal Chemistry. 2011; 88:537552.

[24] Manley D. Biscuit, Cracker and Cookie Recipes for the Food Industry. Woodhead Publishing: Cambridge, UK; 2001.

[25] Delcour JA, Joye IJ, Pareyt B, Wilderjans E, Brijs K, Lagrain B. Wheat gluten functionality as a quality determinant in cereal-based food products. Annual Review of Food Science and Technology. 2012; 3:469-492.

[26] Singh M \& Mohamed A. Influence of gluten-soy protein blends on the quality of reduced carbohydrates cookies. LWT Food Science Technology. 2007; 40:353-360.

[27] Chevallier S, Colonna P, Buleón A, Della Valle G. Physicochemical behaviors of sugars, lipids, and gluten in short dough and biscuit. Journal of Agricultural Food Chemistry. 2000; 48:1322-1326.

[28] Colussi C, Sousa JML, Pinto VZ, Schiavon RA, Elias MC. Propriedades físicas de biscoitos tipo cookie elaborados com farinha de arroz e castanha-do-Brasil, In: Anais do 5o Simpósio Brasileiro de Qualidade de Arroz. Pelotas, Brazil; 2012. Citado em 07 de abril de 2019. Disponível em: https://ainfo.cnptia.embrapa.br/digital/bitstream/item/11561 $1 / 1 / 24923 . \mathrm{pdf}$ 